\title{
A review on pharmacological and analytical aspects of diosgenin: a concise report
}

\author{
Kanika Patel, ${ }^{\mathrm{a}}$ Manoj Gadewar, ${ }^{\mathrm{b}}$ Vijay Tahilyani, ${ }^{\mathrm{c}}$ and Dinesh Kumar PAtel ${ }^{\mathrm{d}, *}$ \\ ${ }^{a}$ G.L.A Institute of Pharmaceutical Research, Mathura, India \\ ${ }^{\mathrm{b}}$ S.K.I.P.S. Warangal, A.P. India \\ ${ }^{\mathrm{c}}$ Sonekar College of Pharmacy, Koradi, Nagpur, India \\ ${ }^{\mathrm{d}}$ Department of Pharmaceutics, Institute of Technology, Banaras Hindu University, Varanasi 221005, India
}

Received 15 February 2012; Accepted 16 March 2012

C The Author(s) 2012. This article is published with open access at Springerlink.com

\begin{abstract}
Diosgenin is a steroidal sapogenin found in plants such as Dioscorea nipponoca, Solanum incanum, Solanum xanthocarpum and Trigonella foenum graecum. Diosgenin, biologically active phytochemicals have been used for the treatment of various types of disorder such as leukemia, inflammation, hypercholesterolemia and cancer. It is also able to prevent bone loss to the same extent as that of oestrogen. It is a typical initial intermediate for synthesis of steroidal compounds, oral contraceptives and sex hormones. Dioscorea, Costus and Trigonella are mainly used for the production of diosgenin. On the basis of literature survey it divulges that diosgenin has very impressive pharmacological profile and could be used as a medicine for the treatment of different types of disorders in the future. Thus, the present work aims to provide collective information in concern with its pharmacological activity and phytoanalytical techniques. This review will be beneficial to researches for the development of an alternative method for the treatment of innumerable diseases from diosgenin.
\end{abstract}

Keywords: analytical techniques, cancer, diosgenin, pharmacological activity, plant source, seperation techniques

\section{Introduction}

Diosgenin (25R-spirost-5-en-3 $\beta$-ol) (Figure 1) is a hydrolysate of dioscin found in the rootstock of yam (Dioscorea) and exists widely in the natural plant in the form of glucoside. ${ }^{1}$ It is a steroidal sapogenin found in various plants such as Dioscorea nipponoca Makino, Smilax china Linn, Smilax bockii Warb, Solanum incanum, Solanum xanthocarpum, Costus speciosus and Trigonella foenum graecum. ${ }^{2,3}$ It is a biologically active phytochemicals responsible for various type of action in the plant including functional food. ${ }^{1}$ It acts as a typical initial intermediate for the synthesis of steroid and contraceptive. However, it is also used in the form of medicine for the treatment of disease like leukemia, hypercholesterolemia, climacteric syndrome and colon cancer. ${ }^{4}$ It is used as initial material for synthesis of oral contraceptives, sex hormones and other steroidal compounds. ${ }^{1}$ In plants it is found in the form of steroidal saponins. Due to its high steroidal saponins content, Dioscorea zingiberensis (C.H. Wright) is used in the China for the production of diosgenin. Acid hydrolysis of the raw herb is the traditional method of diosgenin production where sugar chains of steroidal saponins present in the herb are hydrolyzed to yield diosgenin. ${ }^{5}$ Diosgenin has been proven for the

\footnotetext{
*To whom correspondence should be addressed. E-mail: dkpatel.rs.phe@itbhu.ac.in
}

usefulness in the maintenance of healthy blood cholesterol levels along with the production of dehydroepiandrosterone. Sustained delivery of diosgenin has been able to significantly prevent bone loss. ${ }^{6}$ On the basis of the literature search of diosgenin, it was found that it had very impressive pharmacological profile and could be used as an alternative medicine to treat disparate disorders. Thus, the information summarized in this review will be helpful to the researchers in the field of natural product.

\section{Pharmacological Activity}

\subsection{Effect of Diosgenin against Microorganism}

Diosgenin were investigated for its antimicrobial activity against the human pathogenic yeasts Candida albicans, $C$. glabrata and $C$. tropicalis and found to shown weak antimicrobial activity against all the tested organism.?

\subsection{Effect of Diosgenin against Virus}

Effect of diosgenin on hepatitis $\mathrm{C}$ virus (HCV) replication were evaluated and found to inhibit HCV replication at low micromolar concentrations. However, the levels of viral RNA and viral proteins are also significantly reduced. ${ }^{8}$ 
Effect on immune system
Effect on lipid system
Effect on oxidative system
Effect on inflamatory system

Effect on reproductive system

Effect on cancer

Effect on metabolic process

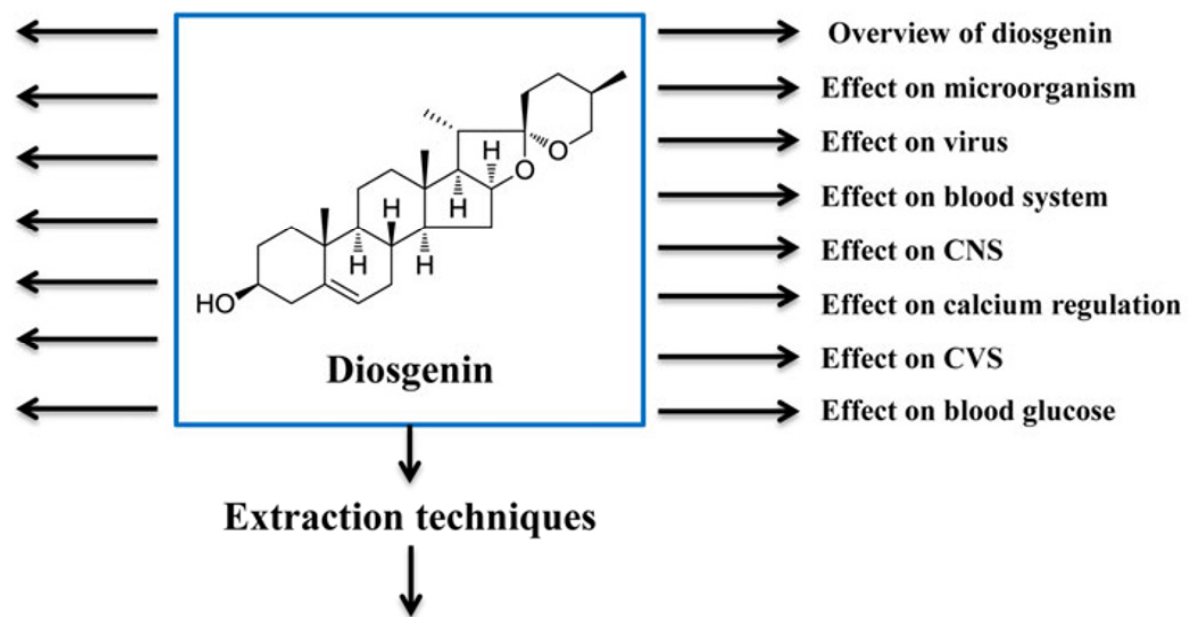

Analytical techniques

Figure 1. Chemical structure and overview of diosgenin

\subsection{Effect of Diosgenin on Blood System}

Anti-thrombosis effect of diosgenin was investigated using an inferior vena cava ligation thrombosis rat model and pulmonary thrombosis mice model both in vitro and in vivo, which resulted with inhibited platelet aggregation, thrombosis and prolonged activated partial thromboplastin time (APTT), prothrombin time (PT) and thrombin time (TT) in rats in a dose-dependent manner. Bleeding time, clotting time is also prolonged along with increased protection rate in mice which revealed it have anti-thrombosis activity. ${ }^{9}$

\subsection{Effect of Diosgenin on CNS}

The action of diosgenin on ion currents in human cortical neurons $(\mathrm{HCN}-1 \mathrm{~A})$ was investigated and the enhanced $\mathrm{BK}$ (Ca)-channel activity was also noted. However, in inside-out patches, diosgenin applied to the intracellular surface showed no effect on $\mathrm{BK}(\mathrm{Ca})$-channel activity. It also elevated intracellular $\mathrm{Ca}^{2+}$ in $\mathrm{HCN}-1 \mathrm{~A}$ cells. Western blotting revealed the presence of the alpha-subunit of $\mathrm{BK}(\mathrm{Ca})$-channels in these cells. Functional activity of cortical neurons may be affected by diosgenin on these channels. ${ }^{10}$ In another study, diosgenin showed significant acetylcholinesterase (AchE) inhibitory activity. ${ }^{11}$

\subsection{Effect of Diosgenin on Calcium Homeostatic System}

Investigation on effect of diosgenin in osteoporosis induce female rats by ovariectomy suggest that bone loss after ovariectomy can be significantly reduced by supplementation with sustained levels diosgenin without jeopardizing other body organs. ${ }^{12}$ Diosgenin up-regulates VEGF-A and promotes angiogenesis in preosteoblast-like cells by a hypoxia-inducible factor-1alpha-dependent mechanism involving the activation of src kinase, p38 MAPK, and Akt signaling pathways via estrogen receptor. ${ }^{13}$ Effect of diosgenin on the osteogenesis of murine MC3T3-E1 osteoblastic cells was investigated. Enhanced bone formation by stimulating the synthesis and secretion of Type 1 collagen and ALP and bone marker proteins Runx 2 and osteopontin expression was observed. The increased levels of these marker proteins can increase the formation of calcium deposition within the ECM thereby increasing bone formation. ${ }^{14}$

\subsection{Effect of Diosgenin on Cardiovascular System}

Many investigations have shown that, diosgenin can significantly reduce the levels of plasma total cholesterol TC and LDL-cholesterol and increase the ratio of HDL-cholesterol to TC by suppressing cholesterol absorption and increasing cholesterol secretion. ${ }^{2}$ Diosgenin as vasorelaxant in superior mesenteric rings were investigated using phenylephrine as a standard and found that it showed concentration-dependent relaxations. A mesenteric endothelial cell loaded with FURA-2 diosgenin has the potential of increasing intracellular calcium concentrations, which was significantly decreased by atropine. In addition, diosgenin also increased nitric oxide (NO) levels. ${ }^{15}$

Effect of diosgenin as vasodilator using porcine resistance left anterior descending coronary artery was investigated and found that it caused an acute endothelium-independent coronary artery relaxation via protein kinase $G$ signaling cascade and an activation of BK (Ca) channel of arterial smooth muscle cells. ${ }^{16}$ Effects of diosgenin on smooth muscle cell contraction and calcium signaling were investigated in the isolated mouse aorta using wire myography and confocal microscopy. Significant blocking of receptor-mediated calcium signals and smooth muscle contraction in the isolated aorta were observed, implying a potential therapeutic value for diosgenin in vascular disorders. ${ }^{17}$

\subsection{Effect of Diosgenin on Blood Glucose System}

The effects of diosgenin on intestinal disaccharidases and some renal enzymes in diabetic rats were investigated for 3 weeks. Diosgenin supplementation significantly decreased lactase and maltase activities compared to the diabetic control group. It also significantly decrease the activity of the transaminases compared to the normal and diabetic control groups. 
Diosgenin demonstrated hypoglycemic properties, which are beneficial in diabetes by reducing intestinal disaccharidases activities. ${ }^{18}$ Effects of diosgenin on fasting blood glucose and intestinal amylase and ATPases in streptozotocin-induced diabetic rats were studied. There was a significant increase in the activity of alpha-amylase in the proximal region of the small intestinal mucosa of diabetic rats treated with diosgenin. In fasting blood glucose, reduced $\mathrm{Na}^{+}-\mathrm{K}^{+}$-ATPase activity, $\mathrm{Ca}^{2+}$ ATPase activity in proximal region compared to the diabetic control was also found. ${ }^{19}$ Effects of diosgenin on enzyme level were investigated. Plasma glucose and glucose6-phosphatase level decreased significantly in diabetic rats fed with diosgenin as compared to the diabetic control. The activities of ATP-citrate lyase, pyruvate kinase and glucose-6phosphate dehydrogenase were significantly reduced in the liver of diabetic rats compared to normal control. ${ }^{20}$ Diosgenin inhibited glucose-uptake in the test along with the inhibition of glucagon-induced HGPa activity in the tested model. ${ }^{21}$

\subsection{Effect of Diosgenin on Immune System}

Suppressive effect of diosgenin on the intestinal $\mathrm{T}$ helper (Th) 2 responses is associated with an enhancement of the regulatory T-cell immunity. Administration of diosgenin markedly attenuated the intestinal expression of interleukin (IL)-4 and GATA3 in Ovalbumin (OVA)-sensitized BALB/c mice. These results suggest that the suppressive effect of diosgenin on allergen-induced intestinal $\mathrm{Th} 2$ responses is closely associated with an up-regulation of the regulatory $\mathrm{T}$-cell immunity in the inflammatory site. ${ }^{22}$ Effect of diosgenin on humoral immunity was investigated in ovalbumin (OVA)sensitized and challenged BALB/c mice for 34 days. The production of OVA-specific serum $\operatorname{IgG} 2 \mathrm{a}$ was significantly enhanced by diosgenin treatment. In parallel with the enhancement of IgG2a, OVA-induced IFN-gamma secretion and mRNA expression were markedly elevated in splenocytes of diosgenin-treated mice. Furthermore, the expression of T-bet, but not of GATA-3, in splenocytes was up-regulated by diosgenin administration. ${ }^{23}$ The effects of three diosgenyl saponins on the immuno stimulating activity in relation to phagocytosis, respiratory burst and nitric oxide production in mouse macrophage cells RAW 264.7 have been investigated. Results showed that diosgenyl saponins significantly enhanced phagocytic activity that increased with the concentration of saponins to reach a maximum, and then tended to decrease with higher concentrations. ${ }^{24}$

Diosgenin were screened for anti-allergic activities using RBL-2H3 cells, the aglycone was found to possess higher activity than the diglucosylated molecule; whereas substitution with rhamnoglucosides apparently results in loss of activity. Furthermore, effects of dioscorealide A, dioscorealide B and dioscoreanone on antigen-induced release of TNF-alpha and IL-4 in the late phase reaction were also examined. ${ }^{25}$ Plant steroid diosgenin causes an inhibition of the growth of fibroblast-like synoviocytes from human rheumatoid arthritis, with apoptosis induction associated with cyclooxygenase-2 (COX-2) up-regulation. These findings suggest that the proapoptotic effect of diosgenin is associated with overexpression of COX-2 correlated with overproduction of endogenous prostaglandin E2. ${ }^{26}$ Effect of incessant deliver diosgenin to adult ovariectomized female rats for 45 days and follow changes in body weight, organ weight and histopathological changes in the adrenal gland were investigated. The results indicated that ovariectomized animals had a significant increase in body weight and spleen weights. Slight increases in wet adrenal weights were observed in the ovariectomized group compared to the control animals. ${ }^{27}$

\subsection{Effect of Diosgenin on Lipid System}

Hypolipidemic effects of diosgenin on rats fed with high cholesterol and high fat diet for 6 weeks was investigated. Diosgenin treatment increased lipoprotein lipase (LPL), hepaticlipase (HL), superoxide dismutase (SOD), glutathione peroxidase (GSH-PX) and nitric oxide synthase NOS activities, thus attenuated oxygen free radicals, decreased malondialdehyde (MDA), TC, TG and LDL-C levels in hyperlipidemic rats. ${ }^{28}$ Hypolipidemic and antioxidative effect of diosgenin on rats fed with a high-cholesterol diet supplemented for 6 weeks were investigated. It showed a decrease in the plasma and hepatic total cholesterol levels, but increased the plasma high-density lipoprotein (HDL) cholesterol level. Furthermore, diosgenin feeding enhanced the resistance to lymphocyte DNA damage caused by an oxidant challenge with $\mathrm{H}_{2} \mathrm{O}_{2}{ }^{29}$ Effects of diosgenin-induced cholesterol hypersecretion on Abcg5/g8 expression in wild-type mice were investigated. Diosgenin did not stimulate biliary cholesterol secretion in mice deficient for Abcg8. Diosgenin feeding increased hepatic expression of genes involved in cholesterol synthesis as well as genes encoding for several cytochrome P450s. ${ }^{30}$

The effect of diosgenin, ethinyl estradiol and this co-administration on changes of lipoprotein metabolism and expression of hepatic genes those are important for cholesterol metabolism including the recently identified abcg 5 and abcg8 in male Wistar rats was invesigated. Diosgenin-feeding induced the hepatic abcg5/abcg8 expressions and biliary cholesterol secretion. ${ }^{31}$ Dioscin and its aglycone, diosgenin, both suppressed the time-dependent increase of blood triacylglycerol level when orally injected with corn oil to mice, suggesting their inhibitory potential against fat absorption. ${ }^{32}$ Diosgenin showed significantly therapeutic and preventive effect on hypercholesterolemia in mice. The serum total cholesterol level was decreased when rats were pre-treated with diosgenin. ${ }^{33}$ Diosgenin inhibited the accumulation of TG and the expression of lipogenic genes in HepG2 cells. Moreover, diosgenin inhibited the transactivation of liver-Xreceptor- $\alpha$, as measured using a luciferase assay system and by gel mobility shift assay. ${ }^{34}$

\subsection{Effect of Diosgenin on Oxidative System}

Effect of diosgenin supplementation on antioxidative enzyme activities were investigated and found to be affected with diosgenin supplementation. Total superoxide dismutase (SOD) in the plasma and liver, glutathione peroxidase (GSH$\mathrm{Px}$ ) in erythrocytes, and catalase (CAT) in erythrocytes and liver were significantly increased in the diosgenin group. The expression of antioxidative enzymes was up-regulated by diosgenin, the expression of GSH-Px being the highest in the diosgenin group. ${ }^{35}$ Effect of diosgenin on the senescent mice induced by d-galactose for sixth week was investigated. It showed increased activities of superoxide dismutase (SOD) and glutathione peroxidase (GSH-Px) and decreased malondialdehyde (MDA) level in the brain of d-galactose treated mice. ${ }^{36}$ Effect of diosgenin for a period of 35 days on

\section{Springer}


lysosomal hydrolases, membrane-bound enzymes, and electrolytes during isoproterenol induced myocardial necrosis in rats was investigated. Diosgenin pretreated groups showed significant decrease in CK-MB, lipid peroxidation, and lysosomal hydrolases activity. Diosgenin pretreatment reversed the membrane-bound enzymes activity and thereby maintained the normal electrolyte concentration, suggesting the protective action in isoproterenol induced myocardial infarction. ${ }^{37}$

\subsection{Effect of Diosgenin on Inflamatory System}

The effect of diosgenin on the expression of adhesion molecules induced by TNF- $\alpha$ in cultured mouse VSMC cell line, MOVAS-1 was investigated, and found that diosgenin inhibits the adhesive capacity of VSMC and the TNF- $\alpha$ mediated induction of ICAM-1 and VCAM-1 in VSMC by inhibiting the MAPK/Akt/NF- $\mathrm{kB}$ signaling pathway and ROS production explaining the ability of diosgenin to suppress inflammation within the atherosclerotic lesion and modulate immune response. ${ }^{38}$ Effects of diosgenin on the production of inflammatory mediators in lipopolysaccharide (LPS)/interferon gamma (IFN-gamma)-activated murine macrophage were investigated. Pretreatment with diosgenin resulted in the inhibition of NO production and inducible nitric oxide synthase (iNOS) protein and mRNA expression in a concentration-dependent manner. ${ }^{39}$

Effect of diosgenin on the inflammatory changes in the interaction between adipocytes and macrophages was investigated. A coculture of 3T3-L1 adipocytes and RAW 264 macrophages markedly enhanced the production of tumor necrosis factor-alpha, monocyte chemoattractant protein-1, and nitric oxide compared with the sum of their single cultures; however, treatment with diosgenin inhibited the production of these proinflammatory mediators. Diosgenin also suppressed the inflammation in RAW 264 macrophages that was induced by the conditioned medium derived from 3T3-L1 adipocytes. ${ }^{40}$ Effect of diosgening on modulating food allergy was investigated in $\mathrm{BALB} / \mathrm{c}$ mice. Diosgenin demonstrated a suppressive effect on the intestinal inflammation, including the occurrence of diarrhea, the infiltration and degranulation of mast cells, and the presence of mucin-containing goblet cells in the duodenum. Furthermore, the serum production of OVAspecific IgE, and the total IgE was suppressed. ${ }^{41}$

\subsection{Effect of Diosgenin on Reproductive System}

An effect of diosgenin on the calpain isoform expression in ovariectomized rats was investigated. To the contrary, no significant difference in the expression of mu- and m-calpain mRNAs among the different diosgenin dosage groups was found. Decreased expression of mu- or m-calpain was observed in the ovariectomized group compared with the normal controls. ${ }^{42}$ Effect of diosgenin on stimulated growth of mammary epithelium for 15 days was investigated with a parameter of increase in DNA content, increase in number of ducts and appearance of terminal endbuds in this study, and the observation was a significant increase in the mammary development scores. Concomitant treatment of estrogen and diosgenin showed augmentation of estrogenic effect of diosgenin especially at the higher dose level. ${ }^{43}$
Effects of diosgenin on cell proliferation induced by IGF-1 in primary human thyroid cells were investigated. Cell viability by MTT and cell proliferation was tested by EdU assay, where as cell cycle analysis was performed by FACS. This study revealed that diosgenin inhibited cell proliferation, caused $\mathrm{G} 0 / \mathrm{G}(1)$ arrest, and could inhibit cell proliferation induced by IGF-1 in primary human thyroid cells. ${ }^{44}$ Diosgenin inhibited migration and invasion of PC-3 cells by reducing matrix metalloproteinase expression. It also inhibited extracellular signal regulating kinase, Jun N-terminal kinase and phosphatidylinositide-3 kinase /Akt signaling pathways as well as NF- $\mathrm{KB}$ activity. These findings reveal new therapeutic potential for diosgenin in anti-metastatic therapy. ${ }^{45}$

Effect of diosgenin on TRAIL-induced apoptosis in HT-29 cells was investigated and found that diosgenin sensitizes HT-29 cells to TRAIL-induced apoptosis. Diosgenin-induced p38 MAPK pathway activation and subsequent DR5 over expression cardinally involve this underlying mechanisms sensitization. ${ }^{46}$ The implication of COX-2 and 5-LOX in diosgenin-induced apoptosis were investigated and results demonstrate how difficult it is to assess the correlation between the apoptotic signaling pathway of diosgenin and arachidonic acid metabolism with certitude. ${ }^{47}$ In another study it was found that kinases including Src, Tor, Akt, CREB, RSK and Chk2 may be implicated in signaling of subsequent apoptosis of mature megakaryocytes after diosgenin-induced differentiation of HEL cells. ${ }^{48}$ In another study, no significant toxicity was seen in the normal breast epithelial cells (MCF10A) following treatment with diosgenin. Additionally, in vivo tumor studies indicate diosgenin significantly inhibits tumor growth in both MCF-7 and MDA-231 xenografts in nude mice. ${ }^{49}$

Implication of cyclooxygenase-2 (COX-2) and thromboxane synthase $(\mathrm{TxS})$ in diosgenin-induced megakaryocytic differentiation in HEL (human erythroleukemia) cells were investigated in another study. ${ }^{50}$ Diosgenin inhibited the reduction of Akt and GSK 3beta phosphorylation induced by LY294, 002, a PI3K inhibitor. Result revealed that production levels of MITF (microphthalmia-associated transcription factor) and tyrosinase were increased by diosgenin. Study showed that diosgenin inhibits melanogenesis by activating the PI3K pathway, and also suggests that diosgenin may be an effective inhibitor of hyperpigmentation. ${ }^{51}$ Food saponin, diosgenin is a potent inhibitor of HCT-116 human colon carcinoma cells by growth inhibition and induction of apoptosis. Importantly, result identifies that the growth suppressive or apoptotic activity of diosgenin may involve cholesterol homeostasis. ${ }^{52}$ Combined association implicating a MEK inhibitor (U0126) and diosgenin is the most effective in inducing very strong apoptosis with down-regulation of COX-2 expression and activity in human RA FLS. ${ }^{53}$ Diosgenin induced megakaryocytic differentiation of HEL cells through a combined activation of ERK and inhibition of the p38 MAPK pathways. Inhibition of ERK activation by a MEK inhibitor abrogated diosgenin-induced differentiation. Afterwards, differentiated cells showed a marked inhibition of expression of survival factors NF-kappaB, Akt and Bcl-xL and activation of caspase-3 together with PARP cleavage leading to apoptotic death of diosgenin-differentiated cells. ${ }^{54}$

Diosgenin decreased DNA binding of NF-kappaB in K562 and HEL cells after 48-h diosgenin treatment. Diosgenin-

\subsection{Effect of Diosgenin on Cancer}

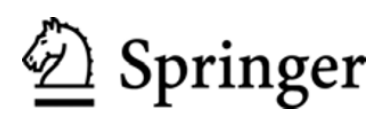


induced apoptosis was associated with cyclo-oxygenase-2 (COX-2) up-regulation in HEL cells but not in K562 cells which are COX-2 deficient. Furthermore, diosgenin inhibited extracellular signal-regulated kinase (ERK) activation only in HEL cells. However, diosgenin activated p38 MAPK in both cell lines and activated c-jun NH2-terminal kinases (JNKs) only in HEL cells. Results suggest that inhibition of NFkappaB nuclear binding and p38 MAPK activation are involved in the diosgenin-mediated signal cascades in K562 cells for inducing/regulating DNA fragmentation. ${ }^{55}$ Diosgenin can effectively inhibit the viability and proliferation of the breast cancer cells. ${ }^{56}$ Diosgenin caused G2/M arrest independently of p53. The levels of cyclin B1 and p21Cip1/Waf1 were decreased, whereas cdc2 levels were increased on the basis of cell cycle analysis. Diosgenin inhibits K562 cell proliferation via cell cycle G2/M arrest and apoptosis, with disruption of $\mathrm{Ca}^{2+}$ homeostasis and mitochondrial dysfunction playing vital roles. ${ }^{57}$

Dietary diosgenin inhibited total colonic aberrant crypt foci (ACF) and multicrypt foci formation in a dose-dependent manner. Diosgenin inhibits cell growth and induces apoptosis in the HT-29 human colon cancer cell line in a dose-dependent manner. ${ }^{58}$ Diosgenin-induced apoptosis is caspase-3 dependent with a fall of mitochondrial membrane potential, nuclear localization of AIF and poly (ADP-ribose) polymerase cleavage. Diosgenin treatment also induces p53 activation and cell cycle arrest in the different cell lines studied. ${ }^{59}$ Diosgenin inhibited S-180, HepA, U 14 mice transplant tumor, the inhibit rates being $30 \%-50 \%$, but it did not inhibit the EAC mice transplant tumor. In vitro, diosgenin inhibited L929, HeLa, MCF cell growth, but it did not significantly affect A375-S2 cells. Diosgenin had an obvious antitumor activity on S-180, HepA, U14 transplant mice in vivo and L929, HeLa, MCF cells in vitro. ${ }^{60}$ Diosgenin treatment caused an inhibition of 1547 cell growth with a cycle arrest in G1 phase and apoptosis induction. Moreover, it was also found a correlation between p53, p21 mRNA expression and nuclear factor kappaB activation and observed a time-dependent increase in PGE2 synthesis after diosgenin treatment. ${ }^{61}$ Diosgenin inhibits receptor-activated nuclear factor-kappaB ligand-induced osteoclastogenesis, suppresses tumor necrosis factor TNFinduced invasion, and blocks the proliferation of tumor cells, all activities known to be regulated by NF-kappaB. The results suggest that diosgenin suppresses proliferation, inhibits invasion, and suppresses osteoclastogenesis through inhibition of NF-kappaB-regulated gene expression and enhances apoptosis induced by cytokines and chemotherapeutic agents. ${ }^{62}$

\subsection{Effect of Diosgenin on Metabolic Process}

The effect of diosgenin on faecal minerals and intestinal lipids in streptozotocin-induced diabetic rats was studied for three weeks. Diosgenin did not altered faecal magnesium, calcium, and zinc excretion but significantly decreased faecal sodium and potassium excretion. It significantly decreased intestinal lipid towards normal, faecal lipids excreted was significantly higher in diabetic rats fed diosgenin. ${ }^{63}$ Effects of dietary diosgenin on indomethacin induced intestinal inflammation and alterations in bile secretion in rats were investigated. Diosgenin pretreatment significantly inhibited weight and food intake decreases and intestinal inflammation. Diosgenin significantly increased biliary cholesterol output and prevented the decreases in bile flow, bile acid output, and biliary alpha-muricholic acid and the increases in biliary hyodeoxycholic acid, deoxycholic acid, and hydrophobicity index of bile. ${ }^{64}$

Effect of diosgenin on bile secretion, focusing on canalicular membrane composition, membrane fluidity, transporter expression, and transporter activity were invetigated. Bile flow, especially bile acid-independent fraction, was increased significantly by diosgenin. Biliary output of $\mathrm{Ch}$ and total glutathione were significantly increased by diosgenin. CMV fluidity and Mrp2 expression were increased by diosgenin. The increase of CMV fluidity and Mrp2 suggests that the choleretic action of diosgenin was based upon both the direct stimulation of transporter expression and indirect transporter activation by an increase of membrane fluidity. ${ }^{65}$ Biliary cholesterol output in rats was stimulated over 3-fold by feeding diosgenin for 5 days, whereas biliary outputs of phospholipid and bile salts were not changed by diosgenin feeding. Isolating and perfusing the liver without bile salts resulted in a rapid and substantial decrease in biliary bile salt output; bile salt depletion abolished the diosgenin-induced increment in biliary cholesterol output, showing that the diosgenin-elevated biliary cholesterol output was bile-saltdependent. ${ }^{66}$

Diosgenin and beta-sitosterol were administered to CRJ/CD male mice for 15 days, the changes in bile acid metabolism was examined. This resulted with some differences between diosgenin and beta-sitosterol in their effects on diet intake, liver weight, and plasma cholesterol level. Most of the increase in fecal excretion of cholesterol occurred 2 days after the start of feeding of phytosterols and gradually declined thereafter, but the levels on day 15 were nevertheless higher than those in the control mice. ${ }^{67}$ Effect of diosgenin in biliary secretion and intestinal absorption of cholesterol in diosgeninstimulated fecal cholesterol excretion in wild type and NPC1L1-knockout (L1KO) mice were investigated. Fecal cholesterol excretion increased in diosgenin-fed WT and $\mathrm{L} 1 \mathrm{KO}$ mice. In an in vitro assay, diosgenin was unable to block NPC1L1-dependent cholesterol uptake. ${ }^{68}$

\section{Extraction Techniques of Diosgenin}

Because of its biological activities, numerous researches on separation and purification of diosgenin from herb have widely been explored through the traditional separation techniques such as liquid-liquid extraction (LLE) and solid-phase extraction (SPE), which can meet the separating requirements of purity. ${ }^{4}$ Methods of extraction of diosgenin from Dioscorea zingiberensis, C.H. Wright include direct acid hydrolysis, spontaneous fermentation, and supercritical $\mathrm{CO}_{2}$ extraction. Recently, some researchers used a single enzyme (such as cellulase and theamylase) combined with acid hydrolysis to treat Chinese yam (Dioscorea opposita Thunb) material, demonstrating that about $70 \%$ diosgenin can be extracted from the material. However, the activity of enzyme gradually reduces owing to the change of catalysis environment, so that the catalysis efficiency of enzyme will also reduce. ${ }^{1}$ A focused microwave-assisted extraction method was developed for the extraction of diosgenin from Fenugreek (Trigonella foenum-graecum) ${ }^{69}$

\section{照 Springer}




\section{Analytical Techniques of Diosgenin}

Gas chromatography-mass spectrometry (GC-MS) method to determine the content of diosgenin in rats has been reported. For quantification; a single-ion monitoring (SIM) mode was used; the limit of quantification (LOQ) was $13 \mathrm{ng} / \mathrm{mL}$, and separation time was $>18$ min. $^{2}$ A sensitive and specific electrospray ionization liquid chromatography-tandem mass spectrometry method was developed to detect diosgenin in the plasma of normal and hyperlipidemic rats. This technique can be used successfully to study pharmacokinetics in rats after oral administration of diosgenin and would be beneficial for the clinical use of diosgenin. ${ }^{70}$

\section{Discussion}

Though, advances have been made in the understanding of the mechanism of action of various herbs, but many questions remain unanswered. For the past few years there has been an exponential growth in the field of herbal medicine and these drugs are gaining popularity both in developing and developed countries because of their natural origin and less side effects. Many traditional medicines in use are derived from medicinal plants, minerals and organic matter. A number of medicinal plants, traditionally used for over 1000 years named rasayana, having their existence in herbal preparations of Indian traditional health care systems. The World Health Organization (WHO) has listed 21,000 plants, which are used for medicinal purposes around the world. Among these 2500 species in India, 150 species are used commercially on a fairly large scale. ${ }^{71-73}$ Because many medicinal plants constitute a rich source of bioactive chemicals that are largely free from adverse effects and have excellent pharmacological actions and could lead to the development of new classes of possibly safer agents for the treatment of disease. Plants have always been an exemplary source of drugs and many of the currently available drugs have been derived directly or indirectly from them. ${ }^{74,75}$

Due to economic constraints, providing modern medical healthcare in developing countries is still a far-reaching goal. The most commonly used drugs of modern medicine such as aspirin, anti-malarial, anti-cancer; digitalis, etc. have originated from plant sources. ${ }^{76}$ Despite the great interest in the development of new drugs to prevent the burden of complications associated with this disease and raised interest in the scientific community to evaluate either raw or isolated natural products in experimental studies, few of them was tested in humans. Based on the available data it was concluded that this bioactive compound have various type of activity and beneficial effect against microorganism, inflammation, cardiovascular disease, blood disorder, cerebral disorder, immune system, oxidative stress, reproductive and cancer chemotherapy. However their specific effects on cancer disease give valuable role for the treatment of various type of cancerous disease. Although phytotherapy continues to be used in several countries, few plants have received scientific or medical scrutinity.

Several medicinal plants have been used as dietary adjunct and in the treatment of numerous diseases without proper knowledge of their function. Only a few herbs and bioactive chemical moiety have attracted the interest of scientists and have been put forward for investigations. However, the pharmacologic actions of these compounds need to be evaluated in studies involving humans to justify the use of these plants or their active principles for the treatment of various disorders. Therefore, there is a need of more well-documented clinical trials and more laboratory work to justify their pharmacological actions and toxicity for safe and effective treatment. Diosgenin which, as per, mentioned above may have all these actions and could prove to be promising in the treatment of various complications especially against cancer in the near future.

In conclusion, the review provides relevant information regarding the pharmacological aspects of diosgenin. The review provides the database of the pharmacological activity of diosgenin, its extraction and analytical procedure for the determination which can be useful for the researcher who wants to explore the hidden potential of this phytoconstituents. The collective information summarized in the review will act as an important segment for development of effective medicines for the treatment of diseases like cancer.

Open Access This article is distributed under the terms of the Creative Commons Attribution License which permits any use, distribution, and reproduction in any medium, provided the original author(s) and source are credited.

\section{References}

[1] Zhang, Y.; Tang, L.; An, X.; Fu, E.; Ma, C. Biochem. Eng. J. 2009, 47, 80-86.

[2] Xu, L.; Liu, Y.; Wang, T.; Qi, Y.; Han, X.; Xu, Y. J. Chromatogr. B 2009, 877, 1530-1536.

[3] Lepage, C.; Léger, D. Y.; Bertrand, J. Cancer Lett. 2011, 301, $193-202$.

[4] Lepage, C.; Léger, D. Y.; Bertrand, J.; Martin, F.; Beneytout, J. L.; Liagre, B. J. Chromatogr. A 2009, 1216, 2254-2262.

[5] Dong, Y.; Teng, H.; Qi, S.; Liu, L.; Wang, H.; Zhao, Y. Biochem. Eng. J. 2010, 52, 123-130.

[6] Yen, M. L.; Su, J. L.; Chien, C. L.; Tseng, K. W.; Yang, C. Y.; Chen, W. F. Mol. Pharmacol. 2005, 68, 1061-1073.

[7] Sautour, M.; Mitaine-Offer, A. C.; Miyamoto, T.; Dongmo, A.; Lacaille-Dubois, M. A. Planta Med. 2004, 70, 90-92.

[8] Wang, Y. J.; Pan, K. L.; Hsieh, T. C.; Chang, T. Y.; Lin, W. H.; Hsu, J. T. J. Nat. Prod. 2011, 74, 580-584.

[9] Gong, G.; Qin, Y.; Huang, W. Phytomedicine 2011, 18, 458-463.

[10] Wang, Y. J.; Liu, Y. C.; Chang, H. D.; Wu, S. N. Planta Med. 2006, $72,430-436$.

[11] Ghayur, M. N.; Kazim, S. F.; Rasheed, H.; Khalid, A.; Jumani, M. I.; Choudhary, M. I. J. Chin. Integr. Med. 2011, 9, 619-625.

[12] Higdon, K.; Scott, A.; Tucci, M.; Benghuzzi, H.; Tsao, A.; Puckett, A. Biomed. Sci. Instrum. 2001, 37, 281-286.

[13] Yen, M. L.; Su, J. L.; Chien, C. L.; Tseng, K. W.; Yang, C. Y.; Chen, W. F. Mol. Pharmacol. 2005, 68, 1061-1073.

[14] Alcantara, E. H.; Shin, M. Y.; Sohn, H. Y.; Park, Y. M.; Kim, T.; Lim, J. H. J. Nutr. Biochem. 2011, 22, 1055-1063.

[15] Dias, K. L.; Correia, Nde A.; Pereira, K. K.; Barbosa-Filho, J. M.; Cavalcante, K. V.; Araújo, I. G.; Silva, D. F. Eur. J. Pharmacol. 2007, 574, 172-178.

[16] Au, A. L.; Kwok, C. C.; Lee, A. T.; Kwan, Y. W.; Lee, M. M.; Zhang, R. Z. Eur. J. Pharmacol. 2004, 502, 123-133.

[17] Esfandiarei, M.; Lam, J. T.; Yazdi, S. A.; Kariminia, A.; Dorado, J. N.; Kuzeljevic, B. J. Pharmacol. Exp. Ther. 2011, 336, 925939.

[18] McAnuff-Harding, M. A.; Omoruyi, F. O.; Asemota, H. N. Life Sci. 2006, 78, 2595-2600.

[19] McAnuff, M. A.; Harding, W. W.; Omoruyi, F. O.; Jacobs, H.; Morrison, E. Y.; Asemota, H. N. Food Chem. Toxicol. 2005, 43, $1667-1672$.

[20] McAnuff, M. A.; Omoruyi, F. O.; Morrison, E. Y.; Asemota, H. N. West Indian Med. J. 2005, 54, 97-101. 
[21] Al-Habori, M.; Raman, A.; Lawrence, M. J.; Skett, P. Int. J. Exp. Diabetes Res. 2001, 2, 91-99.

[22] Huang, C. H.; Liu, D. Z.; Jan, T. R. J. Nat. Prod. 2010, 73, $1033-1037$.

[23] Jan, T. R.; Wey, S. P.; Kuan, C. C.; Liao, M. H.; Wu, H. Y. Planta Med. 2007, 73, 421-426.

[24] Zhang, X. F.; Cui, Y.; Huang, J. J.; Zhang, Y. Z.; Nie, Z.; Wang, L. F. Bioorg. Med. Chem. Lett. 2007, 17, 2408-2413.

[25] Tewtrakul, S.; Itharat, A. Bioorg. Med. Chem. 2006, 14, 87078711.

[26] Liagre, B.; Vergne-Salle, P.; Corbiere, C.; Charissoux, J. L.; Beneytout, J. L. Arthritis Res. Ther. 2004, 6, 373-383.

[27] Benghuzzi, H.; Tucci, M.; Eckie, R.; Hughes, J. Biomed. Sci. Instrum. 2003, 39, 335-340.

[28] Gong, G.; Qin, Y.; Huang, W.; Zhou, S.; Wu, X.; Yang, X. Chem. Biol. Interact. 2010, 184, 366-375.

[29] Son, I. S.; Kim, J. H.; Sohn, H. Y.; Son, K. H.; Kim, J. S.; Kwon, C. S. Biosci. Biotechnol. Biochem. 2007, 71, 3063-3071.

[30] Kosters, A.; Frijters, R. J.; Kunne, C.; Vink, E.; Schneiders, M. S.; Schaap, F. G. Hepatology 2005, 41, 141-150.

[31] Kamisako, T.; Ogawa, H. Hepatol. Res. 2003, 26, 348-352.

[32] Kwon, C. S.; Sohn, H. Y.; Kim, S. H.; Kim, J. H.; Son, K. H.; Lee, J. S. Biosci. Biotechnol. Biochem. 2003, 67, 1451-1456.

[33] Ma, H. Y.; Zhao, Z. T.; Wang, L. J.; Wang, Y.; Zhou, Q. L.; Wang, B. X. China J. Chin. Mater. Med. 2002, 27, 528-531.

[34] Uemura, T.; Goto, T.; Kang, M. S.; Mizoguchi, N.; Hirai, S.; Lee, J. Y. J. Nutr. 2011, 141, 17-23.

[35] Son, I. S.; Kim, J. H.; Sohn, H. Y.; Son, K. H.; Kim, J. S.; Kwon, C. S. Biosci. Biotechnol. Biochem. 2007, 71, 3063-3071.

[36] Chiu, C. S.; Chiu, Y. J.; Wu, L. Y.; Lu, T. C.; Huang, T. H.; Hsieh, M. T. Am. J. Chin. Med. 2011, 39, 551-563.

[37] Jayachandran, K. S.; Vasanthi, H. R.; Rajamanickam, G. V. Mol. Cell Biochem. 2009, 327, 203-210.

[38] Choi, K. W.; Park, H. J.; Jung, D. H.; Kim, T. W.; Park, Y. M.; Kim, B. O. Vascul. Pharmacol. 2010, 53, 273-280.

[39] Jung, D. H.; Park, H. J.; Byun, H. E.; Park, Y. M.; Kim, T. W.; Kim, B. O. Int. Immunopharmacol. 2010, 10, 1047-1054.

[40] Hirai, S.; Uemura, T.; Mizoguchi, N.; Lee, J. Y.; Taketani, K.; Nakano, Y. Mol. Nutr. Food Res. 2010, 54, 797-804.

[41] Huang, C. H.; Ku, C. Y.; Jan, T. R. Planta Med. 2009, 75, 13001305.

[42] Hsu, K. H.; Chang, C. C.; Tsai, H. D.; Tsai, F. J.; Hsieh, Y. Y. J. Obstet. Gynecol. 2008, 47, 180-186.

[43] Aradhana; Rao, A. R.; Kale, R. K. Indian J. Exp. Biol. 1992, 30, 367-370.

[44] Bian, D.; Li, Z.; Ma, H.; Mu, S.; Ma, C.; Cui, B. Arch. Pharm. Res. 2011, 34, 997-1005.

[45] Chen, P. S.; Shih, Y. W.; Huang, H. C.; Cheng, H. W. PloS. One. 2011, 6, 20164.

[46] Lepage, C.; Léger, D. Y.; Bertrand, J.; Martin, F.; Beneytout, J. L.; Liagre, B. Cancer Lett. 2011, 301, 193-202.

[47] Lepage, C.; Liagre, B.; Cook-Moreau, J.; Pinon, A.; Beneytout, J. L. Int. J. Oncol. 2010, 36, 1183-1191.

[48] Cailleteau, C.; Liagre, B.; Beneytout, J. L. J. Cell. Biochem. 2009, 107, 785-796.
[49] Srinivasan, S.; Koduru, S.; Kumar, R.; Venguswamy, G.; Kyprianou, N.; Damodaran, C. Int. J. Cancer 2009, 125, 961967.

[50] Cailleteau, C.; Liagre, B.; Battu, S.; Jayat-Vignoles, C.; Beneytout, J. L. Anal. Biochem. 2008, 380, 26-34.

[51] Lee, J.; Jung, K.; Kim, Y. S.; Park, D. Life Sci. 2007, 81, 249254.

[52] Raju, J.; Bird, R. P. Cancer Lett. 2007, 255, 194-204.

[53] Liagre, B.; Leger, D. Y.; Vergne-Salle, P.; Beneytout, J. L. Int. J. Mol. Med. 2007, 19, 113-122.

[54] Leger, D. Y.; Liagre, B.; Beneytout, J. L. Int. J. Oncol. 2006, 28, 201-207.

[55] Liagre, B.; Bertrand, J.; Leger, D. Y.; Beneytout, J. L. Int. J. Mol. Med. 2005, 6, 1095-1101.

[56] Li, J.; Liu, X.; Guo, M.; Liu, Y.; Liu, S.; Yao, S. Anal. Sci. 2005 $21,561-564$

[57] Liu, M. J.; Wang, Z.; Ju, Y.; Wong, R. N.; Wu, Q. Y. Cancer Chemother. Pharmacol. 2005, 55, 79-90.

[58] Raju, J.; Patlolla, J. M.; Swamy, M. V.; Rao, C. V. Cancer Epidemiol. Biomarkers. Prev. 2004, 13, 1392-1398.

[59] Corbiere, C.; Liagre, B.; Terro, F.; Beneytout, J. L. Cell Res. 2004, 14, 188-196.

[60] Wang, L. J.; Wang, Y.; Chen, S. W.; Ma, J. S.; Fu. Q.; Wang, B. X. China J. Chin. Mater. Med. 2002, 27, 777-779.

[61] Moalic, S.; Liagre, B.; Corbière, C.; Bianchi, A.; Dauça, M.; Bordji, K. FEBS Lett. 2001, 506, 225-230.

[62] Shishodia, S.; Aggarwal, B. B. Oncogene 2006, 25, 1463-1473.

[63] Omoruyi, F. O.; McAnuff-Harding, M. A.; Asemota, H. N. Pak. J. Pharm. Sci. 2006, 19, 269-275.

[64] Yamada, T.; Hoshino, M.; Hayakawa, T.; Ohhara, H.; Yamada, H.; Nakazawa, T. Am. J. Physiol. 1997, 273, 355-364.

[65] Yamaguchi, A.; Tazuma, S.; Ochi, H.; Chayama, K. Hepatol. Res. 2003, 25, 287-295.

[66] Thewles, A.; Parslow, R. A.; Coleman, R. Biochem. J. 1993, 291, 793-798.

[67] Uchida, K.; Takase, H.; Nomura, Y.; Takeda, K.; Takeuchi, N.; Ishikawa, Y. J. Lipid. Res. 1984, 25, 236-245.

[68] Temel, R. E.; Brown, J. M.; Ma, Y.; Tang, W.; Rudel, L. L.; Ioannou, Y. A. Lipid. Res. 2009, 50, 915-923.

[69] Kaufmann, B.; Rudaz, S.; Cherkaoui, S.; Veuthey, J. L.; Christen, P. Phytochem. Anal. 2007, 18, 70-76.

[70] Xu, L.; Liu, Y.; Wang, T.; Qi, Y.; Han, X.; Xu, Y. J. Chromatogr. B 2009, 877, 1530-1536.

[71] Modak, M.; Dixit, P.; Londhe, J.; Ghaskadbi, S.; Devasagayam, T. P. A. J. Clin. Biochem. Nutr. 2007, 40, 163-173.

[72] Patel, D. K.; Kumar, R.; Prasad, S. K.; Hemalatha, S. Asian. Pac. J. Trop. Biomed. 2011, 1, 131-138.

[73] Patel, D. K.; Laloo, D.; Kumar, R.; Hemalatha, S. Asian. Pac. J. Trop. Med. 2011, 4, 748-755.

[74] Patel, D. K.; Prasad, S. K.; Kumar, R.; Hemalatha, S. Asian. Pac. J. Trop. Dis. 2011, 1, 323-329.

[75] Patel, D. K.; Prasad, S. K.; Kumar, R.; Hemalatha. S. Asian. Pac. J. Trop. Biomed. 2012, 2, 320-330.

[76] Grover, J. K.; Yadav. S.; Vats. V. J. Ethnopharmacol. 2002, 81, 81-100. 